\title{
Germanica
}

\section{La Reine des Elfes d'Hubert Lampo : conte philosophique}

Die Elfenkönigin von Hubert Lampo: «philosophisches Märchen»

\section{Gilbert Van de Louw}

\section{OpenEdition}

\section{Journals}

Édition électronique

URL : http://journals.openedition.org/germanica/1347

DOI : $10.4000 /$ germanica. 1347

ISSN : $2107-0784$

Éditeur

Université de Lille

Édition imprimée

Date de publication : 1 janvier 1992

Pagination : 143-155

ISSN : 0984-2632

Référence électronique

Gilbert Van de Louw, «La Reine des Elfes d'Hubert Lampo : conte philosophique », Germanica [En ligne], 11 | 1992, mis en ligne le 07 février 2014, consulté le 06 octobre 2020. URL : http://

journals.openedition.org/germanica/1347 ; DOI : https://doi.org/10.4000/germanica.1347

Ce document a été généré automatiquement le 6 octobre 2020.

(C) Tous droits réservés 


\title{
La Reine des Elfes d'Hubert Lampo : conte philosophique
}

Die Elfenkönigin von Hubert Lampo: «philosophisches Märchen»

\author{
Gilbert Van de Louw
}

1 Lorsque le bourgmestre d'Amsterdam reçut officiellement le roi de Suède en 1923, il fit l'éloge de Selma Lagerlöf, notamment parce qu'elle incarnait le renouveau du merveilleux. Il s'attira, ce faisant, les foudres de Louis Couperus, auteur décadent très en vogue qui lui reprocha vertement d'ignorer la production nationale. L'anecdote illustre, à des titres divers, les difficultés du conte moderne, tout autant que l'éloignement du monde politique et littéraire. C'est déjà l'incommunicabilité qui s'installe et qui va gagner les générations, élargir le fossé entre critiques et public avant de devenir l'enjeu même de la créativité littéraire.

2 Faut-il pour autant tout lui imputer, la toute-puissance de la prose, l'apparent déclin du théâtre dans certaines aires linguistiques, la fuite en avant d'un fantastique toujours plus surprenant? A la réflexion, le vocable signifie surtout le désarroi devant des phénomènes sur lesquels la réflexion théorique traditionnelle -littéraire, philosophique ou historique - n'a plus prise. Il exprime un pessimisme qui, de l'avis quasi général, est né de la guerre, mais dont on saisit mal le fonctionnement par rapport à la réflexion littéraire.

3 Le conte, dans ce contexte, joue un rôle révélateur. Par sa position excentrée dans l'histoire des genres littéraires, ses racines et sources multiples, sa place dans l'inconscient collectif, il devient un observatoire privilégié de cette incommunicabilité qui se saisit de l'étrange pour mieux clamer sa vérité. Observatoire privilégié à cause des apports divers - populaires et littéraires - dont l'antagonisme apparent ou réel explique pour une large part l'indignation de Louis Couperus; observatoire privilégié aussi à cause des glissements structurels : comment établir la différence entre le récit fantastique et le conte ? Observatoire privilégié encore à cause de ce que le conte traditionnel évacuait et que la modernité véhicule : le tragique. Car depuis qu'à l'aube du XXe siècle se posa le problème de la «bonne" guerre, bientôt suivie d'une 
«mauvaise » qui ne s'était point présentée comme telle à tous, la création littéraire se débat dans les contradictions autour de cette tragique révélation.

Dans l'aire néerlandophone qui, rappelons-le, englobe la Hollande et les anciennes Flandres, cette incommunicabilité se nourrit de sensibilités autrement façonnées et de réalités politiques, sociales et culturelles différentes, ne fût-ce que parce que la Belgique a connu la première guerre mondiale, alors que la Hollande est restée neutre. Elle se nourrit d'une vie intellectuelle quasiment antagoniste, dans la mesure où la modernité apparaît plutôt comme l'apanage du Nord, le Sud étant surtout en quête de permanence. Le conte en porte les traces: le Petit Jean (De kleine Johannes) de van Eeden, le Hollandais, traduit en français dès 1908 et parrainé par Romain Rolland, reste la référence première. Mais l'œuvre, insaisissable, se prête peu à un classement définitif, même si on la considère comme symboliste. Au tournant du siècle et ce jusque vers les années 1930, les auteurs vont chercher l'inspiration tantôt dans la spécificité du groupe social ou national, tantôt dans les sources communes de la grande culture grecque notamment. L'œuvre de Couperus présente ainsi plusieurs esquisses - Prélude, par exemple, qui préfigure un conte symbolique de la solitude ${ }^{1}-$ aussi bien que des œuvres achevées tels Fidessa (1899) et surtout Psyché (1898), traduit en français par F. Barbier et publié avec une préface de J. Benda. Le conte apparait tantôt comme une fuite dans la féerie mythologique, tantôt comme le support du merveilleux retour aux sources de la spécificité : c'est le cas de Spookdiligence (Diligence enchantée) de H. van Eyk. Il se présente comme un aimable badinage, autant qu'un mode de réflexion: Godfried Bomans (1913-1971) s'en saisit pour camper les relations entre Hollandais et Flamands ou pour dénoncer les travers d'un monde catholique par trop ultramontain.

5 Il est évident que la nature même du conte nourrit le fantastique comme ce fut le cas des Fantastische Vertellingen (Récits fantastiques) de F. Bordewijk (trois volumes de 1919, 1923, 1924). Devant les modifications qui s'opèrent dans tous les genres littéraires, la frontière reste difficile à tracer entre contes, récits et autres genres où l'on voit fleurir, comme en peinture, le fantastique à travers ce qu'il est convenu d'appeler « le réalisme magique ", dans lequel s'illustrent Bodewijk, J. Daisne et H. Lampo ${ }^{2}$. Ces trois auteurs, par des voies diverses, s'inspirent de l'étrange et du déraisonnable quotidien et renouent, rappelons-le, avec une longue tradition "nordique» qui oppose " perspective » et « optique » et qui se joue de la «narrativité » et du « réalisme » pour avancer sur la voie d'une spécificité « nationale $»^{3}$.

$6 \quad$ Faut-il s'étonner de voir que sur ces trois auteurs, deux sont Flamands? Par ses sujets et sa verve caustique, le conte a connu en Hollande un développement fort différent de ce qu'il fut dans les Flandres où l'on en est resté longtemps au merveilleux d'un Tyl l'Espiègle et au ressourcement à travers l'histoire nationale, comme ce fut le cas pour Symforosa la Béguine de Timmermans (1918)4. Seul L.P. Boon semble être un représentant de la modernité dans ce conte flamand, avec ses grinçants Grimmige Sprookjes. Mais une telle présentation ne tient compte que de l'expression littéraire, de la spécificité du ton et du souffle, alors que le conte moderne polyforme répond à des critères autres que stylistiques, notamment en fonction du merveilleux et du rôle de l'invraisemblable. C'est d'ailleurs à ce niveau que se situent les principales causes de l'incommunicabilité, puisque celle-ci se réfère à un raisonnement qui ne se partage pas ou à une relation verbale qui ne peut plus s'établir, parce que la parole a perdu de sa vérité au profit de la fonction rhétorique. Et qui dit rhétorique dit reconnaissable à partir de procédés 
identifiables. Or, les Métamorphoses de Couperus montrent comment mythe, légende et «fantaisie » se mélangent pour crier l'appréhension devant l'avenir :

o comme nous tremblons !... d'être ceux que nous serons ${ }^{5}$.

7 La peur de l'avenir s'emparant de la création littéraire, que deviendra le conte philosophique? Rappelons que ce genre semble peu prisé de nos critiques qui se réfèrent presqu'uniquement à Voltaire et déduisent de ses œuvres les caractéristiques du genre ${ }^{6}$, notamment la volonté délibérée de détruire l'illusion réaliste, la forme parabolique servant à illustrer un point de vue, avec la présence immodeste du narrateur omniprésent. Mais que deviendra surtout la distanciation qui fait défaut au conte traditionnel?

Hubert Lampo (1920) est un auteur connu pour ses œuvres romanesques, ses essais critiques et la manière dont il aborde les zones inexpliquées de l'histoire littéraire. Ses interprétations n'ont pas l'heur de plaire aux critiques, mais elles ont l'avantage de présenter une solution là où la critique traditionnelle reste muette. Il fait de l'inexplicable l'objet de ses romans et crée un monde à la limite de la réalité et de l'imaginaire où la vérité des personnages se dégage de leur manière d'être devant le mystère de l'existence. On peut donc s'attendre à ce qu'il nous présente dans son œuvre bien des « interfaces » avec le conte, surtout à cause d'un style ample et musical qui, dans des périodes dont on a quelque peu oublié la force persuasive, n'hésite pas à recourir à l'ironie mordante.

$9 \quad$ La Reine des Elfes (De Elfenkoningin, 1989) semble bien relever de ce genre, en dépit de la longueur de l'œuvre - 631 pages - qualifiée de « roman » par l'auteur lui-même. Elle tranche donc singulièrement sur les récits fantastiques et autres courtes nouvelles contemporaines. L'étendue et le souffle épique y soutiennent de multiples déplacements dans le temps et l'espace. Cette dimension permet une lente et longue immersion du lecteur dans un univers hors du temps. Le narrateur est certes omniprésent. Il communique ce qu'il vit, mais ces "aventures» sont de l'ordre extraordinaire : le temps et la raison s'y trouvent remodelés.

10 Le sujet du roman est simple, de tradition partiellement populaire, puisqu'il s'agit d'abord d'un roman d'amour. L'auteur fait une rencontre décisive, tombe éperdument amoureux d'Emily, jeune Anglaise qui est sur le continent pour y mener des recherches littéraires. Cet amour est donc comme un ailleurs linguistique, culturel, affectif, bref, complémentaire. L'auteur en est saisi, alors même qu'il s'occupe à réparer les outrages du temps et à repousser les limites de l'incommunicabilité : il s'intéresse, en effet, à un collègue du XIX $\mathrm{X}^{\mathrm{e}}$ siècle dont il refaçonne l'œuvre pour l'adapter à une sensibilité plus contemporaine. Rappelons qu'en néerlandais bon nombre d'ouvrages devenus illisibles à cause des évolutions de la langue ont été «hertaald», "traduits ", si l'on veut bien accepter ce sens de «traduction » à l'intérieur d'une même langue. L'auteur se présente donc comme un truchement entre deux époques et déterre ainsi un homme que le hasard a affublé du nom de Van Kerkhoven - du Cimetière, en français. Avenir d'un amour partagé et passé collectif se complètent. Le moment est particulièrement important, puisque l'auteur apprend qu'un père qu'il n'avait jamais connu a été abattu dans les derniers jours de la guerre. Tout en travaillant donc sur l'histoire culturelle de son pays, il va à la rencontre de ses propres souvenirs et de son propre manque, tout en se préparant à l'avenir d'un monde partagé.

11 Il façonne donc le passé par le biais de la «traduction » et fait remonter à la surface des éléments perdus de la mémoire collective, ce qui est une autre manière de s'affirmer 
comme Flamand. Mais l'auteur structure ce temps collectif au moment même où son propre passé le déstructure à bien des égards. La division du roman reflète cette complexité puisqu'on dénombre trois parties: Pieter Frans (Pierre-François) du prénom de l'auteur oublié, Emily, chapitres tournés vers l'avenir, Mister Smith, partie consacrée à la découverte de l'assassin du père, mais surtout à l'apprentissage de la distance que la raison fait prendre à partir des sentiments bruts, haine et angoisse.

Les lecteurs de Lampo ont pris l'habitude de ces chasses-croisés où le temps bascule, où les langues se télescopent et les langages s'entrechoquent: le héros de Lampo est en général confronté à l'extraordinaire, soit parce qu'il est éperdument amoureux - et se voit donc jaugé et jugé alors que le monde de ses références habituelles se trouve bousculé -, soit parce que la situation dans laquelle il se trouve ne permet plus aucun repérage logique ou traditionnel. Il est donc prédestiné à une angoisse diffuse mais existentielle.

Ce roman est de guerre et d'amour, c'est la transposition d'éléments populaires dans un univers d'intellectuel. Guerre et amour, le couple est bien étrange, puisque l'un n'empêche guère l'autre, même si la guerre établit un autre ordre autant sur le plan moral que social. Le lecteur voit s'y tisser les fils d'intrigues complexes : la réécriture romanesque, le façonnage linguistique, la découverte d'un père qui n'existe plus, l'affirmation d'un amour qui passe nécessairement par un choix et comporte donc aussi une part de renoncement et de perte. Pour trouver celle que ses amis appellent la Reine des Elfes, le héros tire un trait définitif sur le passé. Il connaitra dans sa vie une faille qui impose un "avant » et un "après » Emily. Aux prises avec son passé, il s'accroche à la vie, à travers ce « sentiment océanique » dont parle Freud, cet amour sans limite, sans frontière, sans fin, ni dans le temps, ni dans l'espace et qui fait d'Emily un prototype. Elle l'aide à surmonter les épreuves du passé : quand il part à la recherche de ceux qui ont tué son père, il découvre les horreurs de la guerre fratricide flamande puisque son père a été abattu dans les derniers jours de la guerre par des S.S. Flamands déchaînés, en pure perte, aucun ordre ne venant plus de la part des Allemands. Dans cette lente descente aux enfers, il découvre un père instituteur, socialiste, libre penseur et francmaçon. Et Lampo, le conteur, de dresser une fresque peu habituelle des divisions de la vie publique belge, avec ses calotins, ses francs-maçons, ses libéraux, ses curés fresque toujours d'actualité. Et à côté des horreurs de cette guerre où les Flamands se battent la rage au cœur, on découvre aussi les chaînes de solidarité ; à côté des sources escamotées, on découvre les bonnes volontés de journalistes qui possèdent l'information et la mettent à la disposition du narrateur, pour lui permettre de mieux saisir une tragédie personnelle ignorée jusque là.

L'atrocité du geste gratuit, la violence qui se nourrit du seul dépit d'avoir parié sur le mauvais cheval, voilà ce que le narrateur découvre. Et le mal gratuit dont l'auteur luimême a connu la variété dans sa propre vie, va être transposée sur le meurtrier du père qui s'avère un ancien élève de l'instituteur. Ce meurtrier, l'auteur découvre finalement que c'est un des criminels qui ont réussi à échapper à la justice des hommes - avec la complicité de l'Eglise catholique, d'ailleurs ${ }^{7}$. Ce meurtrier, finalement, s'avère être le père d'Emily.

Dans l'épilogue, sur le bateau du retour, Emily va dire qu'elle porte en elle une nouvelle vie, celle de leur choix.

Invraisemblable, grotesque, bien sûr. Mais n'est-ce pas par là que la distance se crée et qu'une certaine vérité, au delà des apparences, s'impose? Ce roman n'est pas plus 
invraisemblable que celui des pérégrinations de Candide ou des aventures du Huron, alors même que la notion d'invraisemblable a fortement ébranlé les esprits qui connaissent d'expérience la guerre. Chemin faisant, et grâce à la distanciation romanesque, Lampo brosse une fresque de la vie flamande, où l'histoire et la politique immédiate se rejoignent, où le non-dit impose sa loi et tronque les vérités, avec ses petites lâchetés politiques, ses grands silences officiels, avec une amnistie toujours réclamée et des Flamands d'une nouvelle génération qui croient que la richesse supplée à la mémoire ou efface ce dont personne ne parle plus. Et l'on est heureux de voir enfin se profiler ici l'histoire des Juifs d'Anvers, trop longtemps ignorée, même si le rappel de leurs souffrances est difficile à supporter. Mais sans cet aveu-là, l'honneur ne se recouvre point.

17 Ce conte philosophique nous présente, finalement, une vision du monde à partir de tout ce qui fait l'incommunicabilité, celle du temps et celle des hommes engoncés dans leurs querelles, leur violence et leur lutte pour un pouvoir dont Lampo montre la relativité. L'œuvre se construit autour d'une distante ironie qui ne laisse rien dans l'ombre et s'appuie sur la trame d'un «Bildungs-roman» qui ne dit pas son nom. A partir de là, il n'est guère étonnant de voir que la Reine des Elfes plonge ses racines dans l'invraisemblable: puisque celui-ci n'est plus une référence, dès lors qu'il s'agit des horreurs et de la perversité de l'homme. Le romanesque se mêle à une réalité décrite avec la précision du chroniqueur à travers des images révélatrices, et ce mélange convient au conte philosophique. Il a pu dérouter un public hollandais, habitué à ne point confondre les réalités et les genres et à ériger le thème de la guerre en tabernacle sacro-saint - ce qui est une manière de l'évincer de l'imaginaire collectif. Cet accueil en dit long sur la différence de sensibilité entre Nord et Sud, sur les racines et les limites du « réalisme » hollandais et sur les différences entre Flamands et Hollandais. Pour ces derniers, guerre répond d'abord à paix. C'est l'affaire du citoyen qui ne joue pas avec l'ordre social, alors que l'amour reste circonscrit au jardin secret de l'individu. La production romanesque de ces derniers temps semble prendre cette dimension socioculturelle comme cible, comme en témoigne l'Attentat, roman d'H. Mulisch. Lampo considère, cependant, que la dimension romanesque du conte est indispensable pour aborder le sujet dans son exemplarité, pour boucler la boucle et signifier qu'un pardon suppose toujours un acte gratuit qui fait la richesse de la condition humaine, plus que la raison. C'est l'intérêt de cette œuvre, où s'opposent et se mêlent l'honneur et l'amour, les inexplicables atrocités collectives et les sentiments amoureux que l'on voudrait à l'abri du temps et du temporel. Opposer guerre et amour, c'est pour l'auteur, comme pour les écrivains de la génération de Romain Rolland et d'autres, opposer les " hommes de bonne volonté » aux autres et mettre le lecteur à l'abri d'une rhétorique où la raison deviendrait par trop raisonnable, voire raisonnante. Ces hommes de bonne volonté se révèlent des humanistes, capables d'affirmer leur condition d'homme, sans recours au parapluie pour se protéger des foudres du supérieur, soucieux de suivre l'individu qui leur est confié et de veiller à l'harmonieux développement de sa personnalité. Ce sont, finalement, des hommes et des femmes pour qui «Befehl ist Befehl» n'a pas de sens.

18 Et Lampo d'opposer raison et aliénation collective, par le biais de cette «bonne volonté " d'une part et la "répétition» de l'autre. Car dans le comportement de beaucoup de Flamands de l'époque, il trouve une absence de raisonnement, prélude à l'absence de morale, tout simplement, parce que dans la répétition l'individu s'aliène. L'incommunicabilité née d'une dépendance répétitive éloigne la raison. Et Lampo 
montre dans une des nombreuses digressions un vieillard hébété, avec des accès de folie douce, perdu dans le monde contemporain : il croyait à son engagement dans les S.S., mais se refusait à la perversion et aux abus collectifs. Mais son devenir est symptomatique d'une raison qui fait naufrage, puisqu'elle ne se soutient plus de la morale. Folie et perversion, voilà les deux écueils, car la répétition aliène et ne laisse à celui qui s'y soumet qu'un seul exutoire: celui de la perversion et de l'agressivité gratuite, signe de l'exclusion dont le meurtrier porte les marques : il est celui qui est marqué du sceau de la Bête. On le reconnaît par delà les temps et les changements à un énorme anthrax que le dictionnaire définit comme « tumeur inflammatoire affectant le tissu cellulaire sous-cutané et qui se termine toujours par la gangrène ». " Neuf yeux " (Negenoog) en néerlandais, cette marque associe la morale au visuel - et nous sommes encore dans un monde où l'optique prévaut sur la perspective, puisque cette optique a un sens que le français a égaré depuis longtemps 8 .

19 Si conte philosophique il y a, quel est donc son sens? On le découvre à travers les différents thèmes de l'œuvre, parmi lesquels celui de la culture et de l'enracinement affectif - aussi bien personnel que collectif. Seulement, le relevé des thèmes ne suffit pas. L'œuvre devient, par ce qu'elle est, représentative de ce que le conte entend illustrer - d'où l'importance de cette révélation bien flamande d'un passé ignoré jusque là. Rappelons, pour ce qui est de la culture, que dans les années 1940 encore, une revue hollandaise comme De Stem, pouvait poser la question de savoir si la guerre faisait partie de la culture 9 . Lampo s'attache à faire de ses héros des personnages éminemment culturels : souvent ils sont journalistes, auteurs, chercheurs, bref ils occupent des postes pour lesquels l'indépendance de l'esprit est primordiale. Il les inscrit ainsi dans un questionnement sur l'ordre culturel, par trop dévoyé dans le monde actuel. Cet univers est pour lui d'abord un espace où les idées s'affrontent, mais où les échanges n'entraînent pas de ruptures. C'est dire que la violence s'exprime sans excès, que la raison reste maître du jeu et du lieu. Or, il constate que le monde de l'éducation flamande, de l'université, de la critique et de la création littéraire sont par trop façonnés par les modes exclusives et pas assez par les convictions.

D'où des renvois à des situations cryptées dont le lecteur ne saisit peut-être pas toujours la portée, mais dont il comprend l'ironie mordante. Et ces modes sont relayées par l'appareil politique qui ne trouve pas davantage grâce aux yeux de l'auteur. Mais ne sommes-nous pas en face de la transposition littéraire d'une réalité qui apparaît bel et bien ainsi à l'extérieur?

L'ancrage dans la culture et dans le lieu affectif, voilà deux éléments qui déterminent l'attitude du narrateur. La culture faite de raison et d'intelligence reflète la pétillance de l'esprit créateur et de l'ironie mordante de l'observateur qui ne se veut pas dupe. Le lieu affectif, c'est plus compliqué à définir ; certes, c'est la Flandre ou plutôt le pays d'Anvers pour lequel Lampo a une affection particulière : c'est vers Anvers, plus que vers la Belgique ou les Flandres que s'en retournent le narrateur et Emily à la fin de leur voyage. Mais le lecteur est tiraillé entre la Flandre et l'Angleterre, pays de naissance d'Emily et refuge du père. Et dès que se pose le problème de l'ancrage, force est de constater que l'auteur joue sur deux plans : celui de la femme venue d'ailleurs et donc mythique, celui de ses propres origines affectives. L'Angleterre fait comprendre cette dichotomie entre affect et sentiment, car c'est le pays où les cimetières sont particuliers :

Une fois passé la Manche, c'est certainement le cas... on s'imagine difficilement des endroits plus paisibles. La mort est ce à quoi on pense en tout dernier lieu... ${ }^{10}$. 

démocratique. Cette Angleterre que Lampo connaît bien pour s'être fortement intéressé aux nombreuses péripéties du roi Arthur ${ }^{11}$, devient un lieu où vie et mort se rejoignent. Et c'est à travers ces notions de vie et de mort que la philosophie de ce conte apparaît le mieux. Rappelons que, dans les pays du Nord, ces vocables ont toujours gardé une connotation morale - et de ce point de vue, il n'est pas étonnant de voir une filiation entre cette Reine des Elfes et la pansophie de Coménius - cette volonté de tout subordonner à l'unité de l'homme et de ramener la vie à ce qui est de l'ordre de la nécessité. La langue de Coménius est métaphorique à souhait et le lecteur de Lampo se voit confronté à une langue et une construction romanesque où la métaphore prend la place de la parodie voltairienne, tout simplement, parce que l'homme ne s'affirme plus de la même manière : il sait trop quels sont les manques de sa condition, combien il est davantage fait de questionnements que de solutions, combien il est peu lui-même sans le complément que l'autre constitue. D'où l'intérêt de ces va-et-vient entre l'Angleterre et le continent que l'on appelle en néerlandais « la terre ferme », comme si l'aventure amoureuse ramenait le héros à la terre ferme, à une unité, une complétude après un pari pascalien, bref après une excursion dans l'inconnu de l'autre dont il reconnaît manquer. Cet abandon d'une part de soi-même et le don gratuit qui en est la conséquence, les héros l'inscrivent dans leur itinéraire géographique et culturel, dans les lieux et l'espace vital. L'aventure amoureuse révèle au narrateur la nécessité de l'intemporel. La langue en devient un témoignage. La Reine des Elfes montre des personnages qui incarnent métaphoriquement le bien et le mal et leur attitude face à la langue devient révélatrice : Emily fait des recherches en littérature néerlandaise, qui est pour elle le domaine de l'altérité. Mister Smith agit autrement vis-à-vis de la langue, parce que le don n'est pas dans sa nature et que donc il n'a pas de prise sur le culture. Car à travers la langue, c'est la culture qui s'exprime. Et ce conte montre que la guerre a été faite par des abrutis qui n'ont pas le sens de la culture. Ils connaissent, certes, fort bien la musique allemande, mais confondent culture et savoir, âme et technique. Et dans cette opposition entre technique et morale, on retrouve bien encore le testament de Coménius qui ne s'exprime pas autrement dans Unum necessarium (1668), quand il fustige les hommes qui ne savent distinguer l'accessoire de l'essentiel. Pour Lampo aussi, seule une culture humaniste porte remède à la perversion du regard technique, parce que l'humilité seule jugule la violence tout en l'exprimant. Cette culture, elle intègre la distanciation intellectuelle, comme le montre surtout l'usage de la langue. Lampo fait partie de cette génération d'auteurs pour qui la langue néerlandaise est un lieu mythique auquel il apporte sa propre contribution par une prose très musicale qui n'a guère changé, en dépit des modes qui rapetissent et «objectivent " la phrase. Il connaît le prix à payer pour façonner sa sensibilité dans une langue qui demeure pour une part une acquisition. Et cette langue est l'enjeu de bien des difficultés en Belgique, pour les Wallons, comme pour les Flamands qui luttent contre «l'excursion dans l'inconnu " par un retour au dialecte. Dans ce contexte, il est donc fort révélateur de voir ce que Lampo fait de la langue par rapport à ce Monsieur Smith que le narrateur traque et trouve mourant. Cet homme a pu, dans les milieux aristocratiques où il évoluait, tromper son monde sur l'accent qui était le sien, en prétextant un long séjour dans les camps nazis où il aurait été amené à la suite des événements de Dunkerque. Il maîtrise de nombreuses langues, mais sur son lit de mort, face au fils de l'instituteur, il retombe dans le dialecte anversois, source de référence et unique ancrage culturel. Et 
cela contraste fortement avec Emily qui parle néerlandais avec un délicieux accent, qui est la marque d'un long cheminement vers la découverte de l'autre.

On découvre, finalement, que le sens de ce conte philosophique, c'est peut-être de cultiver son jardin, mais alors de le faire sans plus se voiler la face, sans avoir peur, surtout. Et la modernité de ce conte apparaît bien dans cette angoisse dont le conte philosophique ne peut plus faire l'économie. Lampo s'inscrit ainsi dans la lignée de Couperus, même s'il doit s'étonner de se trouver en pareil voisinage. L'angoisse explique qu'il s'agit d'un conte où les personnages évoluent dans l'invraisemblance pour mieux mettre en évidence des réalités historiques occultées et un avenir qui ne se trace plus depuis les horreurs de la guerre. Angoisse et incommunicabilité se manifestent dans la fonction dévolue au temps: le narrateur déterre son auteur; il découvre les horreurs de la guerre et les ravages du temps collectif. Mais il tourne la page en toute connaissance de cause, parce qu'une aventure amoureuse l'a amené à toucher à des nécessités humaines hors du temps et à considérer l'impérieuse nécessité d'un avenir qui s'inscrit dans sa volonté, comme dirait Coménius, c'est-à-dire dans la faculté de construire un monde conforme à ce à quoi il croit. Et c'est par cette dimension atemporelle que ce roman rejoint encore le conte philosophique, même si c'est au détriment du personnage d'Emily que le lecteur ne voit qu'à travers le narrateur, c'est-à-dire à travers un regard amoureux, qui laisse peut être peu de place à la réalité de celle qui nous est présentée comme héroöne, plutôt que comme individu.

\section{NOTES}

1. - Cf. pour ces différents éléments Frédéric Bastet, Louis Couperus Een biografie, Amsterdam, Querido, 1987, notamment p. 497 pour ce qui est de Prélude. Les rapports de Couperus et du conte pourraient, d'ailleurs, faire l'objet d'une intéressante étude, car ils n'ont pas été très poussés jusqu'à présent.

2. - Rappelons que Lampo a pris une part très active dans le développement de cette notion et que son apport est fort original : plutôt que de faire en sorte que la prose - et les mots - sécrètent cette étrangeté qui est à l'origine du « réalisme magique », il utilise les phénomènes de l'étrange pour créer les œuvres. Il installe les «archétypes » dans l'univers romanesque et s'appuie sur Jung pour expliquer cette étrangeté, explication qui mériterait un examen plus approfondi. En effet, il semble bien que l'angoisse est à l'origine de cette œuvre et que l'explication pourrait tout aussi bien en être freudienne.

3. - Cf. à ce propos Svetlana Alpers, De Kunst van het kijken (L'Art de regarder), titre original, The Art of describing, University of Chicago, 1983, et notamment chap. 2 «Uta pictura, ita visio».

4. Timmermans a exploité cette veine jusqu'en 1948, date à laquelle apparaît encore de sa main en collaboration avec Antoon Thiry - un recueil de récits intitulé : Begynhofsproken.

5. Louis Couperus, Verzameld Werk, IX, «De herscheppingen» in Legende, mythe en fantazie, p. 666-673, p. 673 «o hoe huiveren wij!... te zijn, die zullen zyn...». C'est sur ces mots que le récit se termine.

6. - Cf. J.-P. de Beaumarchais, D. Couty, A. Rey, Dictionnaire des littératures de langue française, Bordas, 1984, article signé M. Simonsen. 
7. - De Elfenkoningin, p. 407-409.

8. - Rappelons les résultats de E. Panowsky sur l'optique qui, dans la sensibilité moderne a perdu son sens philosophique pour devenir une branche de l'activité scientifique.

9. - De Stem, XX, 4 avril 1940, Dr W. van Ravesteyn, «Maakt de oorlog deel uit van de "cultuur"?», p. 353-366.

10. - Eenmaal het Kanaal over wél... Men kan zich onmogelijk vrediger plekjes voorstellen. Het laatste waar je aan denkt is de dood... Elfenkoningin, p. 138.

11. - Cf. le téléfilm « Sur les traces du roi Arthur » et le livre correspondant.

\section{RÉSUMÉS}

Unis dans une même langue, Hollandais et Flamands n'en sont pas moins tributaires de conditions et valeurs socio-culturelles divergentes. Le conte - et plus particulièrement le conte philosophique - de part et d'autre de la frontière nationale trouvera, face à la modernité et au lancinant problème de la guerre, des expressions littéraires différentes. Hubert Lampo est un auteur dont l'éclairage du "réalisme magique » est fort original. Sa Reine des Elfes apparaît comme une œuvre qui relève du conte philosophique moderne, intégrant dans la démarche créatrice l'angoisse, l'incommunicabilité et l'invraisemblance. Ces éléments font partie d'une quête personnelle du héros qui apprend à les subordonner à une démarche culturelle dans laquelle l'enracinement, les choix linguistiques et la quête de savoir font contrepoids à une morale faite d'obéissance et de répétition. Dans cette quête, le héros découvre le poids du groupe et les vicissitudes de l'histoire. Plutôt que de "cultiver son jardin", il décide, en toute connaissance de cause, de parier sur la vie et de vivre d'amour, parce qu'il a découvert le besoin qu'il a de l'autre.

Durch eine und dieselbe Sprache verbunden, sind Holländer und Flamen nichtsdestoweniger von grundverschiedenen Verhältnissen und soziokulturellen Werten abhängig. Das Märchen insbesondere das philosophische - erhält diesseits und jenseits der nationalen Grenze im Verhältnis zur Modernität und zur quälenden Kriegsfrage unterschiedliche literarische Ausdrucksformen. Der Autor Hubert Lampo beleuchtet den «magischen Realismus» auf recht eigenartige Weise. Seine Elfenkönigin bietet sich als ein Werk dar, das in die Gattung des «philosophischen Märchens» gehört und dabei beim Schöpfungsprozeß Angst, Kommunikationsunvermögen und Unwahrscheinlichkeit mit einbezieht. Solche Elemente gehören zur persönlichen «Suche» des Helden, der es lernt, sie einem Kultur orientierten Benehmen unterzuordnen, in welchem Verwurzelung, Auswahl der Sprachmittel und Wissensdrang eine auf Gehorsam und Wiederholung beruhende Moral ausgleichen. Bei dieser Suche entdeckt der Held die Bedeutung der Gruppe und die Wechselfälle der Geschichte. Anstatt «seinen Garten zu bebauen», entschließt er sich bei Kenntnis der Sachlage, vielmehr auf das Leben zu setzen, aus der Liebe heraus zu leben, und zwar, weil er eingesehen hat, daß er des Anderen bedarf. 
AUTEUR

GILBERT VAN DE LOUW

Université Charles-de-Gaulle - Lille III 